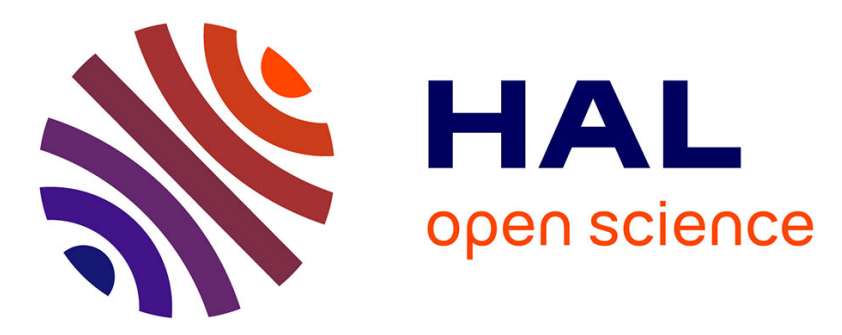

\title{
Pair wave functions for strongly correlated fermions and their determinantal representation
}

\author{
J.P. Bouchaud, A. Georges, C. Lhuillier
}

\section{To cite this version:}

J.P. Bouchaud, A. Georges, C. Lhuillier. Pair wave functions for strongly correlated fermions and their determinantal representation. Journal de Physique, 1988, 49 (4), pp.553-559. 10.1051/jphys:01988004904055300 . jpa-00210729

\section{HAL Id: jpa-00210729 https://hal.science/jpa-00210729}

Submitted on 1 Jan 1988

HAL is a multi-disciplinary open access archive for the deposit and dissemination of scientific research documents, whether they are published or not. The documents may come from teaching and research institutions in France or abroad, or from public or private research centers.
L'archive ouverte pluridisciplinaire HAL, est destinée au dépôt et à la diffusion de documents scientifiques de niveau recherche, publiés ou non, émanant des établissements d'enseignement et de recherche français ou étrangers, des laboratoires publics ou privés. 


\title{
LE JOURNAL DE PHYSIQUE
}

J. Phys. France 49 (1988) 553-559

AVRIL 1988, PAGE 553

Classification

Physics Abstracts

$67.50-74.20-74.70$

Short Communication

\section{Pair wave functions for strongly correlated fermions and their determinantal representation}

\author{
J.P. Bouchaud $\left({ }^{1}\right)$, A. Georges $\left({ }^{2}\right)$ and C. Lhuillier $\left({ }^{1,3}\right)$
}

$(1)$ Laboratoire de Spectroscopie Hertzienne de l'ENS, 24 rue Lhomond, 75005 Paris, France

2 Laboratoire de Physique Théorique de l'ENS, 24 rue Lhomond, 75005 Paris, France

(3) Physique Théorique des Liquides, Université Pierre et Marie Curie, 4 place Jussieu, 75005 Paris, France

(Reçu le \& janvier 1988, accepté le 9 février 1988)

Résumé.- Nous montrons que l'amplitude de toute fonction BCS à nombre de particules fixé est un déterminant. Ceci à une importance numérique considérable et permet une approche variationnelle de ces fonctions, appliquée à l'hélium-3 liquide ou au modèle de Hubbard. Nous proposons d'autre part un nouveau type de fonction d'onde, décrivant un état mixte composé de fermions appariés et célibataires. Les propriétés physiques de cet état sont suggérées.

Abstract. - We show that a (Jastrow projected or not) BCS wave function with any fixed number of fermions can be represented as a determinant. This is important for their numerical investigation, with applications to liquid $\mathrm{He}^{3}$ and Hubbard's model. We also propose a new type of wave function, describing a mixed state composed of paired and unpaired fermions. The physical properties of such a state are tentatively discussed.

\section{Introduction.}

Most approaches to $N$-fermion systems are based on an antisymmetrical wave function (wf) made of individual orbitals arranged in a Slater determinant : this is the case of e.g. the shell model in atomic and nuclear physics, the Gutzwiller w-f for Hubbard's model, the Jastrow-Slater function for $\mathrm{He}^{3}$, etc. Usually, one further assumes weak coupling or Landau's adiabaticity ansatz for Fermi liquids and chooses as individual orbitals the three fermion orbitals (or some distorted versions of them to improve variational calculations). However, a notorious counter-example of non-Slater fermionic w-f is given by the B.C.S. theory of superconductivity which takes as basic entities pair wave functions and build with them a correctly antisymmetrized state. Those pair correlations handle 
the attractive interaction between fermions more efficiently.

Until recently, one only considered B.C.S. wave functions which differed only weakly from plane-wave Slater determinants : all the action usually takes place within a thin energy shell $\left(\sim T_{\mathrm{c}} / E_{\mathrm{f}}\right)$ around the Fermi-surface.

However, for strongly interacting fermionic systems, one should a priori exploit the full variational character of the B.C.S. wave functions and let the momentum distribution $N(k)$ adopt its optimal shape, which may be quite far from the usual Heavyside step and in fact may not satisfy perturbative requirements such as the Migdal-Luttinger theorem imposing a discontinuity of $N(k)$ at $k_{\mathrm{F}}^{0}=\left(3 \pi^{2} \rho\right)^{1 / 3}$.

This possibility has recently been independently suggested for high $T_{\mathrm{c}}$ superconductors [2] and for liquid $\mathrm{He}^{3}$ [3], leading to a new description of the normal state as a non superfluid, strongly paired liquid, for which the superfluid transition and the pairing correlations are characterized by two very different energy scales : it is quite possible to have strong pair correlations while off-diagonal long range order or phase rigidity cannot establish due e.g. to the importance of hard cores heavily suppressing density fluctuations [2]. In order to test this idea, numerical approaches (such as variational calculations) are vital, and some encouraging results have been obtained on the singlet RVB pair w-f (for Hubbard's model) $[4,5]$ and on the triplet Jastrow-BCS w-f for liquid $\mathrm{He}^{3}$ [3]. Those numerical approaches are nevertheless $a$ priori difficult, because of the $N$ ! terms present in these antisymmetrized pairs $w-f$. The aim of this note is to show that all BCS type w-f (including RVB or Jastrow-projected BCS) can be given a determinantal representation. This allows numerical calculations as easily as for Slater-type w-f and could also help to make some analytical progress on e.g. Nagaoka-BrinkmannRice bandwidth calculations $[6,7,8]$.

In a second part, which is more speculative, we propose to generalize the B.C.S. $w$-f to describe a mixed-state where some fermions are paired and the remaining fraction left in individual orbitals, while preserving global antisymmetry. This $\mathrm{w}-\mathrm{f}$ can be given a determinantal representation as well. We briefly speculate on the physics of such a state, which perhaps gives some variational content to the Heavy-Boson model proposed by Ranninger et al. [9] to describe heavy fermions and $\mathrm{He}^{3}$.

\section{The fixed number of particles B.C.S. function.}

One usually write the B.C.S. $w-f$ in a grand canonical ensemble representation, for which the number of particles is not kept fixed. For the usual singlet states, one has (see e.g. [10])

$$
\Psi_{\mathrm{BCS}}=\prod_{k}\left(u_{k}+v_{k} a_{k \uparrow}^{+} a_{-k \downarrow}^{+}\right)\left|O>=\mathrm{e}^{\sum \varphi_{k} a_{k \uparrow}^{+} a_{-k \downarrow}^{+}}\right| O>
$$

with $\varphi_{k}=\frac{v_{k}}{u_{k}}$

Projecting on the state with exactly $N$ particles yields $(P=N / 2$ being the number of pairs)



The free gas Slater-determinant is simply obtained by setting $\varphi_{k}=1$ if $k<k_{\mathrm{f}}$ and $\varphi_{k}=0$ otherwise.

The real space representation of this paired w-f is obtained -by a Fourier transform of (2.2) as :

$$
\Psi_{\mathrm{BCS}}^{N}\left(\mathbf{r}_{1} \ldots \mathbf{r}_{N}\right)=A[\tilde{\varphi}(12) \tilde{\varphi}(34) \tilde{\varphi}(56) \ldots]
$$

where $A$ stands for the total antissymmetrizer and $\tilde{\varphi}$ is an odd spin-orbital w-f, which reads, in the singlet state :

$\left({ }^{1}\right)$ In all that follows, we deliberately omit overall phase factors and normalizations, which are of no interest for our purpose. 


$$
\tilde{\varphi}(12)=\frac{1}{\sqrt{2}} \varphi\left(\mathbf{r}_{1}-\mathbf{r}_{2}\right) \mid 1 \uparrow 2 \downarrow-1 \downarrow 2 \uparrow>
$$

in which $\varphi(\mathbf{r})$ is the even orbital function, simply related to $\varphi_{k}$ by a Fourier transform.

The triplet states can also be represented by (2.3), with :

- for $M_{\mathbf{S}}=0$

$$
\begin{aligned}
& \tilde{\varphi}(12)=\frac{1}{\sqrt{2}} \varphi\left(\mathbf{r}_{1}-\mathbf{r}_{2}\right) \mid 1 \uparrow 2 \downarrow+1 \downarrow 2 \uparrow> \\
& \varphi(\mathbf{r}) \text { odd }
\end{aligned}
$$

- and for $M_{\mathrm{S}}= \pm 1$

$$
\begin{aligned}
& \tilde{\varphi}(12)=\varphi\left(\mathbf{r}_{1}-\mathbf{r}_{2}\right) \mid 1 \uparrow 2 \uparrow>(\text { or } \mid 1 \downarrow 2 \downarrow>) \\
& \varphi(\mathbf{r}) \text { odd }
\end{aligned}
$$

For example, for 4 particles, (2.3), reads :

$$
\Psi^{4}=[\tilde{\varphi}(12) \tilde{\varphi}(34)-\tilde{\varphi}(13) \tilde{\varphi}(24)+\tilde{\varphi}(14) \tilde{\varphi}(23)]
$$

In order to carry variational calculation, one needs to calculate average values $\langle\Psi|O| \Psi\rangle=\bar{O}$ of operators (Hamiltonian, spin correlations, identity (norm)).

The standard Monte-Carlo method takes as a complete basis for the Hilbert space, the set of positions of the fermions, say $\left\{R_{i}^{\uparrow}\right\}_{i=1 \ldots P}$ for the up spins and $\left\{R_{j}^{\dagger}\right\}_{j=1 \ldots P}$ for the downs spins.

$\bar{O}$ is then obtained in the form :

$$
\bar{O}=\sum_{\substack{\left\{R_{i}^{\dagger}\right\}\left\{R_{j}^{\dagger}\right\} \\\left\{\bar{R}_{i}^{\dagger}\right\}\left\{\bar{R}_{j}^{\dagger}\right\}}}\left\langle\Psi \mid\left\{R_{i}^{\dagger}\right\}\left\{R_{j}^{\dagger}\right\}\right\rangle\left\langle\left\{R_{i}^{\dagger}\right\}\left\{R_{j}^{\dagger}\right\}|0|\left\{\bar{R}_{i}^{\dagger}\right\}\left\{\bar{R}_{j}^{\dagger}\right\}\right\rangle\left\langle\left\{\bar{R}_{i}^{\dagger}\right\}\left\{\bar{R}_{j}^{\dagger}\right\} \mid \Psi\right\rangle
$$

One thus needs to compute, for each generated configuration, the amplitude $\left\langle\left\{R_{i}^{\uparrow}\right\}\left\{R_{j}^{\dagger}\right\} \mid \Psi\right\rangle$ for the choosen w-f. We shall now show that this amplitude is not as hard to calculate as (2.3) might suggest, since it can be written as a $p \times p$ determinant, thus computable in $p^{3}$ steps. Note that if one chooses a Jastrow (or Gutzwiller) projected BCS w-f (such as an RVB state), the amplitude is simply multiplied by some configuration dependent reduction factor, which in no way spoils the usefulness of this determinantal representation.

3. Amplitudes as determinants.

3.1 THE $M_{\mathrm{s}}=0$ CASE (SINGLET OR TRIPLET). - For pair wave functions of the type $(2.4,5)$, we will now show the following results :

$$
\left\langle\left\{\mathbf{R}_{i}^{\dagger}\right\},\left\{\mathbf{R}_{j}^{\downarrow}\right\} \mid \Psi\right\rangle=\operatorname{Det} M_{i j}
$$

with $M_{i j}=\varphi\left(\mathbf{R}_{i}^{\uparrow}-\mathbf{R}_{j}^{\downarrow}\right)$

The proof is quite straightforward using (2.2) as a starting point : indeed, one has : 


$$
\begin{aligned}
& \left\langle\left\{\mathbf{R}_{i}^{\dagger}\right\},\left\{\mathbf{R}_{j}^{\dagger}\right\} \mid \Psi\right\rangle=\sum_{k_{1} \ldots k_{p}} \varphi_{k_{1}} \ldots \varphi_{k_{p}}\left\langle\left\{\mathbf{R}_{i}^{\dagger}\right\}\left|a_{k_{1 \dagger}}^{+} \ldots a_{k_{p \dagger}}^{+}\right| O\right\rangle\left\langle\left\{\mathbf{R}_{j}^{\dagger}\right\}\left|a_{-k_{1 \downarrow}}^{+} \ldots a_{-k_{p \downarrow}}^{+}\right| O\right\rangle \\
& =\sum_{k_{1} \ldots k_{p}} \varphi_{k_{1}} \ldots \varphi_{k_{p}} \frac{1}{\sqrt{p !}} \operatorname{Det}\left[\mathrm { e } ^ { i k _ { \alpha } R _ { \beta } ^ { \dagger } ] } \frac { 1 } { \sqrt { p ! } } \operatorname { D e t } \left[\mathrm{e}^{\left.-i k_{\alpha} R_{\beta}^{\dagger}\right]}\right.\right. \\
& =\sum_{k_{1} \ldots k_{p}} \varphi_{k_{1} \ldots \varphi_{k_{p}}} \frac{1}{p !} \sum_{\sigma \sigma^{\prime}} \epsilon_{\sigma} \epsilon_{\sigma^{\prime}} \prod_{\alpha} \mathrm{e}^{i k_{\alpha}\left[\boldsymbol{R}_{\sigma(\alpha)}^{\dagger} \boldsymbol{R}_{\sigma^{\prime}(\alpha)}^{\dagger}\right]}
\end{aligned}
$$

(using the very definition of a determinant).

So

$$
\left\langle\left\{\mathbf{R}_{i}^{\dagger}\right\},\left\{\mathbf{R}_{j}^{\downarrow}\right\} \mid \Psi\right\rangle=\frac{1}{p !} \sum_{\sigma \sigma^{\prime}} \epsilon_{\sigma^{\prime}} \epsilon_{\sigma} \prod_{\alpha} \varphi\left(\mathbf{R}_{\sigma(\alpha)}^{\dagger}-\mathbf{R}_{\sigma^{\prime}(\alpha)}^{\downarrow}\right)
$$

Reindexing the sum with $\sigma^{\prime \prime}$-replacing $\sigma^{\prime}$ - such that $\sigma^{\prime}(\alpha)=\sigma^{\prime}(\sigma(\alpha))$, one has

$$
\frac{1}{p !} \sum_{\sigma \sigma^{\prime \prime}} \epsilon_{\sigma}\left(\epsilon_{\sigma^{\prime \prime}} \epsilon_{\sigma}\right) \prod_{\alpha} \varphi\left(\mathbf{R}_{\sigma(\alpha)}^{\downarrow}-\mathbf{R}_{\sigma^{\prime \prime}(\sigma(\alpha))}^{\downarrow}\right)=\frac{1}{p !} \sum_{\sigma} \operatorname{Det} \varphi\left(\mathbf{R}_{\sigma(\alpha)}^{\dagger}-\mathbf{R}_{\sigma(\beta)}^{\downarrow}\right)=\operatorname{Det}\left[M_{i j}\right]
$$

hence the result (3.1) [5]. Note that the usual Slater determinants of plane waves can also be written in the form (3.1). Indeed, the free fermions ground state is given by (2.2) with $\varphi_{k}=1$ if $k<k_{\mathrm{f}}$ and zero otherwise. The same algebra thus immediatly yields :

$$
\left.\begin{array}{l}
\Psi_{\text {free }}=\operatorname{Det}\left[\varphi_{0}\left(\mathbf{R}_{i}^{\dagger}-\mathbf{R}_{j}^{\downarrow}\right)\right] \\
\text { with } \varphi_{0}(\mathbf{R})=4 \Pi \int_{0}^{k_{\mathfrak{f}}} k^{2} \frac{\sin k R}{k R} \mathrm{~d} k
\end{array}\right\}
$$

Thus some kind of pair correlations do exist in the free gas w-f, characterized by a slow oscillating decay with distance. When attraction is switched on, a bound state appears, and thus a lenght scale $\xi$ governing the exponential decrease of $\varphi$ for large $R$.

3.2 CALCUlation OF OPERATOR AVERAGES.- Using (3.1), one can easily express e.g. the norm $\langle\Psi \mid \Psi\rangle$ as a sum over configurations $C$ :

$$
\langle\Psi \mid \Psi\rangle=\sum_{C=\left\{R_{i}^{\dagger}, R_{j}^{\dagger}\right\}}\left[\operatorname{det} \varphi\left(R_{i}^{\dagger}-R_{j}^{\downarrow}\right)\right]^{2}
$$

Assume for definiteness that we deal with Hubbard's model. Then $\left\{R_{i}^{\dagger}, R_{j}^{\downarrow}\right\}$ are restricted to take discrete values, and the kinetic energy reads $K=-t\left(\Sigma_{\langle i j\rangle} C_{i}^{+} C_{j}(<i j\rangle\right.$ means that the sum is restricted to nearest neighbours). In this case, one has :

$$
\langle\Psi|K| \Psi\rangle=-t \sum_{C} \sum_{i} \operatorname{det} \varphi(C) \operatorname{det} \varphi\left(C_{i}^{\prime}\right)
$$

where $C^{\prime}{ }_{i}$ is a configuration obtained from $C$ by moving atom $i$ by one lattice spacing.

More interesting is the mean value of the spin correlation function

$$
\left\langle\Psi\left|\sum_{i j} \mathbf{S}_{i} \mathbf{S}_{j} \delta\left(R-R_{i j}\right)\right| \Psi\right\rangle=\rho_{S}(R)
$$

Writing $2 \mathbf{S}_{i} \mathbf{S}_{j}=P_{i j}^{\chi}-\frac{1}{2}$ where $p_{i j}^{\chi}$ permutes the spins of atoms $i$ and $j$, one obtains : 


$$
\left\langle\Psi\left|\sum_{i j} P_{i j}^{\chi} \delta\left(R-R_{i j}\right)\right| \Psi\right\rangle=\sum_{C,(i, j)=}[\operatorname{det} \varphi(C)]^{2} \delta\left(R-R_{i j}\right)-\sum_{C,(i, j) \neq} \operatorname{det} \varphi(C) \operatorname{det} \varphi\left(P_{i j} C\right) \delta\left(R-R_{i j}\right)
$$

where $\Sigma_{(i, j)}=$ denotes the sum over identical spins and $\Sigma_{(i, j) \neq}$ over reversed spins, and $P_{i j} C$ is the configuration obtained from $C$ by permuting $R_{i} \uparrow$ and $R_{j} \downarrow$.

3.3 THE $\left|M_{\mathrm{S}}\right|=1 \mathrm{CASE}$ (EQUAL SPIN PAIRING). - In this case we have obtained the slightly weaker result (which we have used in [3] for our numerical calculations) :

$$
\begin{aligned}
& \left\langle\left\{\mathbf{R}_{i}^{\dagger}\right\},\left\{\mathbf{R}_{j}^{\downarrow}\right\} \mid \Psi\right\rangle^{2}=\operatorname{Det} M_{\alpha \beta}^{\dagger} \times \operatorname{Det} M_{\alpha \beta}^{\downarrow} \\
& \text { with }\left\{\begin{array}{l}
M_{\alpha \beta}^{\dagger}=\varphi\left(\mathbf{R}_{\alpha}^{\dagger}-\mathbf{R}_{\beta}^{\dagger}\right) \\
M_{\alpha \beta}^{\downarrow}=\varphi\left(\mathbf{R}_{\alpha}^{\downarrow}-\mathbf{R}_{\beta}^{\downarrow}\right)
\end{array}\right\}
\end{aligned}
$$

Note the uncertainty in the sign of the amplitude, which prevents from calculating, the offdiagonal elements of the 1-particle density matrix.

Working directly with (2.3), one notices that the spin part of the w-f completly factorizes, leaving to evaluate

$$
A[\varphi(12) \varphi(34) \ldots \varphi(p-1, p)] \equiv A \varphi
$$

Now, this is equal to a Gaussian integral over Grassmann variables $z_{i}[11]$

$$
\begin{aligned}
& \left(\left[z_{i}, z_{j}\right]_{+}=0, \int \mathrm{d} z_{i}=0, \int \mathrm{d} z_{i} z_{i}=1\right): \\
& A \varphi \equiv \frac{1}{\left[\frac{p}{2}\right] !} \quad \int \mathrm{d} z_{1} \ldots \mathrm{d} z_{p} \mathrm{e}^{\sum_{i j} z_{i} \varphi_{i j} z_{j}}
\end{aligned}
$$

This can easily be seen by expanding the exponential in powers of $\varphi$. Only the terms of order $\varphi^{P / 2}$ survive the integration over the $z_{i}$ 's and the anticommutation rules. Those terms appear with the correct sign thanks to these anticommutation rules. It is a standard result of Grassmannian algebra that the integral (3.4) is a Gaussian representation of the square root of a determinant [11]:

$$
\int \mathrm{d} z_{1} \ldots \mathrm{d} z_{p} \mathrm{e}^{\sum z_{i j} \varphi_{i j} z_{j}} z_{i} \varphi_{i j} z_{j} \propto \pm \sqrt{\operatorname{Det} \varphi}
$$

This leads, up to a normalization of $\Psi$, to the result (3.3).

4. A new variational wave function : "the fermion-boson mixed state".

4.1 PRESENTATION.- it is quite tempting to think that, for a generic interaction potential, a $\mathrm{w}-\mathrm{f}$ for which part of the atoms are paired and part of them left in individual states could be favoured as compared to the usual BCS state : indeed, the variational space is thereby considerably enlarged (the fraction of paired fermions is a new variational parameter) and improvement appears compulsory. New interesting physics seem to be contained in such a w-f, which we describe more formally before commenting on. Let $\tilde{\varphi}$ be the $M_{\mathrm{S}}=0$ pair w-f of paragraphe 3 and $\tilde{\theta}_{\alpha}$ some one particle w-f characterized by $\alpha$ (e.g. plane waves of wave vector $k_{\alpha}$, or localized states around impurities, etc.). Then, the $N$-body $w$-f which describes our unpolarized fermion-boson mixed state $\left({ }^{2}\right)$ reads :

$\left({ }^{2}\right)$ As for the BCS w-f there are no true bosonic entities in this description : the fermionic symmetry of the basic entities is strictly built in. 


$$
\Psi=A\left[\tilde{\varphi}(12) \ldots \tilde{\varphi}(M-1, M) \tilde{\theta}_{1}^{\dagger}(M+1) \ldots \tilde{\theta}_{\frac{N-M}{2}}^{\downarrow}(N)\right]
$$

It is possible to show that the amplitude of $\Psi$ is also a determinant (of dimension $N-M / 2$ ), thus allowing numerical investigations of this new state :



$$
\begin{aligned}
& p+\frac{N-M}{2}
\end{aligned}
$$

where the unprimed numbers are associated with the $R_{i}^{\dagger}$ and the primed ones with $R_{i}^{\downarrow}$.

4.2 Physical Content.- Suppose that the optimal momentum distribution is found to have the shape drawn in figure 1 , meaning that $\theta$ 's are plane waves with wave vectors less than or equal to $k^{*}$. The ground state of the fermionic assembly would thus contain a mixture of pairs and of normal atoms. (In a sense, a certain number of spinons [2] are present in the ground state). Such a state would exhibit Fermi liquid like behaviour (linear specific heat, constant susceptibility, etc.) characterized by a Fermi momentum which is not dictated by the liquid density as it is the case in Landau's canonical theory. Such a ground state can be unstable against Cooper pairing of the solitary fermions, which undergo their own BCS transition at a temperature not a priori related to the energy scale of the preexisting pairs $\left({ }^{3}\right)$. This possibility is extremely interesting with regard to $\mathrm{He}^{3}$, since it allows us to reconcile our variational results [3] suggesting strong pair correlations, and the familiar Landau description of its low temperature properties $\left({ }^{4}\right)$. We thus propose that the ground

$\left({ }^{3}\right)$ The symmetry of the spinons pair $w-f$ is also a priori different from that of the preexisting pairs.

(4) We cannot of course exclude the existence of a weak discontinuity of $N(k)$ at the $k_{\mathrm{F}}^{0}=$ $\left(3 \pi^{2} \rho\right)^{1 / 3}$. What we really mean is that, in a strong coupling regime, the Pauli principle is ensured through proper pair antisymmetrization, and has no reason to impose anything special at $k_{\mathrm{F}}^{0}$. state structure of liquid $\mathrm{He}^{3}$ has the exotic nature, realizing a true non-adiabatic, strong coupling Landau-Fermi liquid.

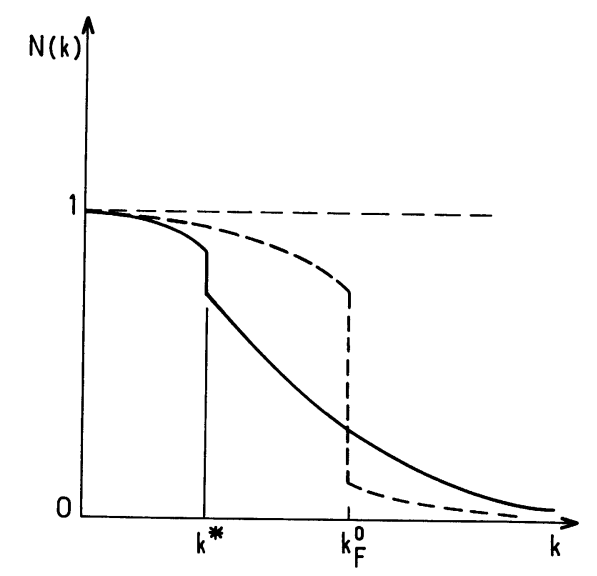

Fig. 1. - Momentum distribution of an interacting Fermi liquid : (a) dashed line : canonical Landau-Fermi liquid, with its Migdal-Luttinger discontinuity at $k_{\mathrm{F}}^{0}$; (b) solid line : distribution expected for a mixed state, with a discontinuity at shifted $k^{*}$ (corresponding to the density of unpaired fermions), around which quasiparticle excitations may develop.

\section{Conclusion.}

In this paper, we have shown that the absolute value of the amplitude of any fixed number of particles B.C.S. w-f (singlet or triplet) can be expressed as determinants. We feel that this result is of some importance for more analytical 
and numerical work on those wave functions, especially in the strong coupling regime.

We have also proposed a new variational family of w-f which contains both the $B C S$ branch and the standard Fermi liquid as limits and which again can be calculated as determinants. These $w-f$ allow us to consider an intriguing state for fermions made of paired atoms and unpaired entities. A crucial question is to know whether such a state exists, with liquid $\mathrm{He}^{3}$ as a good possible candidate.

\section{References}

[1] see e.g. LANDAU-LifSHITz, Statistical Mechanics, part II (Pergamon Press).

[2] ANDERson, P. W., Science 235 (1987) 1196;

Baskaran, G., Zou, Z., ANderson, P. W., Commun. Solid. State Phys. 63 (1987) 973;

ANDERSON, P. W., BASKaraN, G., ZoU, Z., Hsu, T., Phys. Rev. Lett. 58 (1987) 2790;

ANDERSON, P. W., Lect. given at Varenna (July 1987).

[3] Bouchaud, J. P., Lhuillier, C., Europhys. Lett. 3 (1987) 1273.

[4] Yokoyama, H., Shiba, H., J. Phys. Soc. Jpn. 56 (1987) 3570.
[5] Gross, C., Preprint. Note that this author also obtained result (3.1).

[6] NAgAoKA, Y., Phys. Rev. 147 (1966) 392.

[7] Brinkmann, W. F., Rice, T. M., Phys. Rev. B 2 (1970) 1324.

[8] Heritier, M., J. Phys. France 48 (1987) 1849.

[9] RANNINGER, J., ROBASZKIEWICZ, S., SUlPICE, A., TOURNIER, R., Europhys. Lett. 3 (1987) 347.

[10] LeggetT, A. J., Rev. Mod. Phys. 47 (1975) 331

[11] Berezin, F. A., The Method of Second Quantization (Academic Press, N.Y.) 1965. 